Original Article

\title{
Influence of clozapine on neurodevelopmental protein expression and behavioral patterns in animal model of psychiatric disorder induced by low-level of lead
}

\author{
Hwayoung Lee ${ }^{1}$, Minyoung Lee ${ }^{1}$, Hyung-Ki Kim', Young Ock Kim², Jun-Tack Kwon', and Hak-Jae Kim ${ }^{1, *}$ \\ 'Department of Clinical Pharmacology, College of Medicine, Soonchunhyang University, Cheonan 31151, ${ }^{2}$ Department of Bio-Environmental Chemistry, \\ College of Agriculture and Life Sciences, Chungnam National University, Daejeon 34134, Korea
}

\section{ARTICLE INFO}

Received March 30, 2019

Revised August 26, 2019

Accepted October 8, 2019

*Correspondence

Hak-Jae Kim

E-mail: hak3962@sch.ac.kr

\section{Key Words}

Animal model

Behavior rating scale

Clozapine

Lead poisoning

Neurodevelopmental disorder

\begin{abstract}
Exposure to lead during pregnancy is a risk factor for the development of psychiatric disorders in the offspring. In this study, we investigated whether exposure to low levels of lead acetate $(0.2 \%)$ in drinking water during pregnancy and lactation causes behavioral impairment and affects the expression of proteins associated with neurodevelopment. Lead exposure altered several parameters in rat offspring compared with those unexposed in open-field, social interaction, and pre-pulse inhibition tests. These parameters were restored to normal levels after clozapine treatment. Western blot and immunohistochemical analyses of the hippocampus revealed that several neurodevelopmental proteins were downregulated in lead-exposed rats. The expression was normalized after clozapine treatment $(5 \mathrm{mg} / \mathrm{kg} /$ day, postnatal day 35-56). These findings demonstrate that downregulation of several proteins in leadexposed rats affected subsequent behavioral changes. Our results suggest that lead exposure in early life may induce psychiatric disorders and treatment with antipsychotics such as clozapine may reduce their incidence.
\end{abstract}

\section{INTRODUCTION}

Lead is a nonessential, toxic environmental contaminant and lead exposure and accumulation triggers various acute and chronic effects in humans [1,2]. Lead poisoning is associated with the most severe consequences during brain development, including differentiation, intense cellular proliferation, and synaptogenesis [3]. Acute exposure to lead during prenatal and postnatal periods is associated with psychosis, intellectual deficits, and juvenile delinquency [4-6]. Moreover, lead exposure during early life affects embryonic development. It is associated with negative outcomes including reduced neurocognitive development $[7,8]$. These effects manifest as deficits in social interaction, working memory, and spatial learning $[9,10]$. In addition, lead exposure impairs cognitive function and induces chronic changes in hip- pocampal and hypothalamic neurogenesis [11]. It also suppresses neuronal differentiation in central nervous system, inhibits longterm potentiation, inhibits the secretion of neurotransmitters, and interferes with calcium signaling [12]. All these processes affected by lead exposure are essential for neuronal function, synaptic plasticity, transmission of neurite growth, synaptogenesis, and axonal transport, which are required to maintain an intact microtubule structure [13]. Prenatal lead exposure affects embryonic development. It is also associated with negative outcomes in humans from birth to adulthood [7]. In addition, exposure to low levels of lead during early brain development impairs cognitive functions in children [14]. It also affects various cognitive domains, including attention, executive function, and social behavior [15]. Prepulse inhibition (PPI) is an operational parameter in sensorimotor gating. It has been hypothesized that PPI reflects (i) (8) This is an Open Access article distributed under the terms of the Creative Commons Attribution Non-Commercial License, which permits unrestricted non-commercial use, distribution, and reproduction in any medium, provided the original work is properly cited. Copyright $\odot$ Korean J Physiol Pharmacol, pISSN 1226-4512, elSSN 2093-3827
Author contributions: Authors H.J.K. designed and directed the entire project. H.L., M.L. were managed the literature searches and analyses. Authors H.K.K. and J.T.K. performed most of the statistical analyses, Y.O.K. performed edited most of the table and figure data, and asistanted statistical analyses. H.L. contributed substantially to the first draft of the manuscript. All authors contributed to, and have approved, the final manuscript. 
the ability to filter extraneous interoceptive and exteroceptive stimuli $[16,17]$. Reduced PPI has been observed in patients with schizophrenia and related disorders [16,17]. It has been hypothesized that reduced PPI leads to impaired cognition reflecting gating of sensory input to the brain [18]. Several animal studies have demonstrated that PPI deficits can be induced by administration of dopamine agonists [19], NMDA antagonists [20], and exposure to low levels of lead [14]. Clozapine is an atypical antipsychotic agent and a synthetic dibenzodiazepine derivative, which inhibits several neurotransmitter receptors in the brain [21]. Moreover, clozapine was related to sensorimotor gating function that was restored in PPI and the mean\% PPI scores after clozapine treatment of the maternal immune activation-induced schizophrenia animal model [22].

However, the effects of clozapine on lead-induced psychiatric disorders in an animal model of neurodegeneration in early life have yet to be reported. Therefore, the present study was designed to further characterize the pharmacological mechanisms of clozapine in psychiatric disorders in an animal model of prenatal lead exposure. Specifically, the present study determined the effect of clozapine on PPI modulation in startle, open-field, and social interaction tests using an animal model with chronic lowlevel lead exposure via oral route. Subsequently, we measured the protein exposure related to neurodevelopment in the hippocampus area via western blot and immunohistochemical analyses.

Schizophrenia is a neurodevelopmental disorder with a complex etiology comprising both genetic and environmental factors. Prenatal stress or maternal exposure induces cognitive deficits in offspring [23]. The cognitive deficits in schizophrenia are induced by disrupting the hippocampal anatomy and the function of hypothalamic-pituitary-adrenal axis [24,25]. Dihydropyrimidinaselike 2 (Dpys12) is a neurodevelopmental protein that regulates axonal outgrowth via promotion of microtubule, vesicle trafficking, and synaptic physiology in the developing brain [23-25]. Moreover, Dpysl2 associated with disrupted in schizophrenia 1 (DISC1) interacts with many other proteins involved in synaptic function and neurodevelopment pathways related to schizophrenia and depression $[23,26]$. Postsynaptic density protein 95 (PSD95) is an essential component involved in glutamatergic transmission, synaptic plasticity, and dendritic spine morphogenesis during neurodevelopment. Interestingly, PSD-95 binds directly to DISC1 in high-risk schizophrenia and is responsible for synapse formation and microtubule network dynamics $[24,27]$.

\section{METHODS}

\section{Animals and drugs}

Sprague-Dawley female rats were purchased from Daehan Biolink, Ink (Eumsung, Korea) and reared at the animal facility of our institution. All animal procedures were performed in ac- cordance with the guidelines for the care and use of laboratory animals of the United States National Institutes of Health [28]. The study protocol was approved by the Institutional Animal Care and Use Committee (IACUC) in Soonchunhyang University (Korea, IACUC approval No. SCH16-0059). Rats were provided free access to food and water. Pregnant females were individually housed in maternity cages on Gestation day 1. Lead acetate solution $(0.2 \%)$ was prepared in tap water. Glacial acetic acid $(0.5 \mathrm{ml} /$ $\mathrm{L}$ ) was added to lead acetate solution to prevent precipitation. Rats in the test group were exposed to $0.2 \%$ lead acetate in drinking water throughout the gestation and lactation periods (a total of 42 days). No mortality of rat dams was observed at weaning [20]. Clozapine (Sigma-Aldrich Co., St. Louis, MO, USA) was dissolved in $0.1 \mathrm{~N} \mathrm{HCl}$ to a concentration of $5 \mathrm{mg} / \mathrm{kg} /$ day. The animals were orally treated with clozapine solution during stress for 21 days. All groups contained litters of 7 to 12 pups with an equal number of males and females. Extremely large or small litters were eliminated. Offspring were weaned 23 days after birth and housed in groups. Male offspring were selected and used for further experiments. A total of three experimental groups of adolescents were tested: 1) control group comprising offspring of normal mothers; 2) LEAD group including offspring of mothers subjected to lead exposure before parturition; 3) LEAD-CLZ group, with clozapine-treated offspring in the adolescence period. Each group comprised 13 male rats.

\section{Behavioral tests}

Modified behavioral tests, including social interaction test (SIT), open-field test (OFT), and prepulse inhibition test (PPI) were performed as described previously [29-31].

\section{Prepulse inhibition (PPI)}

An automated startle reflex system (SR Lab, San Diego Instruments, San Diego, CA, USA) was used to measure PPI. The system consisted of a startle chamber housed in a sound attenuated isolation cabinet equipped with an internal fan and light. A cylindrical, transparent, acrylic holding apparatus resting on a fourpegged platform within the isolation chamber was used to hold each subject throughout the testing session (subject age, 57 days old). PPI is defined as the percent decrease in startle amplitude as a function of the magnitude of prepulse stimulus using the following formula: percent decrease $=100-\{[($ startle response for prepulse + pulse trial) / (startle response for pulse alone trial)] $\times$ $100\}$. Mean \% PPI was calculated based on the total prepulse test levels at 3, 6, and $12 \mathrm{~dB}$ above background [31].

\section{Open field test (OFT)}

OFT was used to assess the exploratory activity and reactivity to a novel environment. Subjects were removed from their home 
cages on test day (subject age, 58 days) and placed individually in an open-field start box $(15 \times 15 \times 20 \mathrm{~cm})$ for $5 \mathrm{~min}$. The apparatus was constructed with black Polygal $(77 \times 77 \times 25 \mathrm{~cm})$. No background noise was detected. The experimenter exited the room and the subjects behavior was recorded for $20 \mathrm{~min}$ [29-31].

\section{Social interaction test (SIT)}

SIT was adapted from previous studies and conducted in a black Polygal chamber $(77 \times 77 \times 25 \mathrm{~cm})$ (subject age, 59 days). The room in which the chamber was located was darkened during testing. The chamber was illuminated by a single $25 \mathrm{~W}$ red light bulb placed $\sim 100 \mathrm{~cm}$ above the base of the chamber. Social interaction partners were same-sex siblings that resided in the same cage after weaning. They had approximately equal body weights. Each session lasted $20 \mathrm{~min}[30,31]$.

\section{Western blot}

Hippocampus tissues were lysed ( $\mathrm{N}=5-6$ animals per group) in RIPA buffer (EBA-1149; Elpis-Biotech, Inc., Daejeon, Korea) containing protease inhibitors and centrifuged at 14,000 rpm for $10 \mathrm{~min}$ at $4^{\circ} \mathrm{C}$. To detect Dpysl 2 and neurofilament protein $\mathrm{M}, 200 \mu \mathrm{g}$ of lysed protein was subjected to 10 and $12 \%$ sodium dodecyl sulfate-polyacrylamide gel electrophoresis (SDS-PAGE) and transferred to PVDF membranes (Millipore, Milford, MA, USA). After blocking with 5\% skim milk, the membranes were incubated with anti-Dpysl2 (1:1,000; Cell Signaling Technology, Danvers, MA, USA), neurofilament M (Nefm; 1:1,000; Cell Signaling Technology), PSD95 (Dlg4; 1:1,000; Cell Signaling Technology), and $\beta$-tubulin (Tubb; 1:3,000; Thermo Fisher Scientific, Inc., Rockford, IL, USA) antibodies overnight at $4^{\circ} \mathrm{C}$ followed by probing with peroxidase-conjugated secondary anti-mouse (1:10,000; Sigma-Aldrich Co.) and secondary anti-rabbit (1:5,000; Abfrontier, Seoul, Korea) antibodies for $1 \mathrm{~h}$ at room temperature. Immunoreactive bands were detected using an enhanced chemi-
A

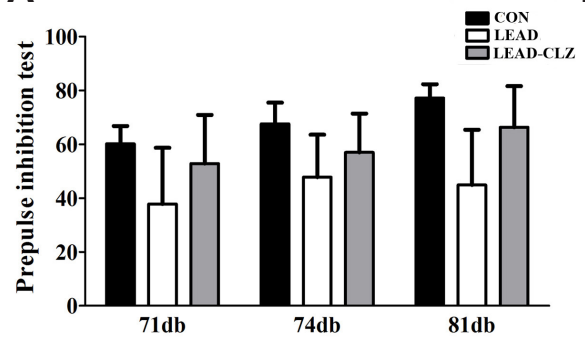

B

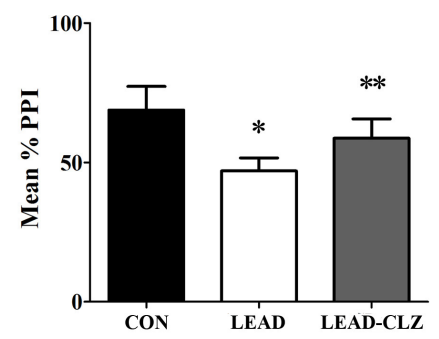

Fig. 1. Prepulse inhibition (PPI) in adult male rats was analyzed on post-natal day 56 after exposure to lead stress during gestation or in male rats derived from unstressed litters during gestation. (A) The prepulse at intensities of 3, 6 and $12 \mathrm{~dB}$ were applied above the background noise preceded the onset of the $120 \mathrm{~dB}$ pulse. (B) The mean percent PPI values. Each group comprised 10 male rats. Each bar represents the mean of 10 male rats ( \pm standard deviation); ${ }^{*} p<0.05$ compared to the control group; ${ }^{* *} p<0.05$ compared to clozapine treatment group; LEAD, lead-exposed offspring during prenatal and lactation periods; CON, non-lead exposed offspring; LEAD-CLZ, lead-exposed and clozapine-treated offspring.

Table 1. Behavior of non-lead exposed group, lead exposed group and lead exposure with clozapine treatment for adolescence in a social interaction test

\begin{tabular}{|c|c|c|c|}
\hline Actions & $\mathrm{CON}$ & LEAD & LEAD-CLZ \\
\hline Sniffing $(n)^{+}$ & $68.33 \pm 5.91$ & $68 \pm 11.81$ & $115.11 \pm 6.80$ \\
\hline Sniffing $(s)^{+}$ & $83.44 \pm 10.39$ & $75.22 \pm 9.37$ & $200.67 \pm 15.57$ \\
\hline Following $(n)^{+}$ & $20.56 \pm 4.31$ & $30.22 \pm 4.99$ & $16.22 \pm 2.44$ \\
\hline Following $(\mathrm{s})^{*}$ & $38.67 \pm 6.93$ & $60.89 \pm 10.53$ & $51.11 \pm 8.46$ \\
\hline Grooming to the patner $(n)^{*,+}$ & $3.56 \pm 1.86$ & $0.22 \pm 0.15$ & $3.22 \pm 1.02$ \\
\hline Grooming to the patner $(\mathrm{s})^{*,+}$ & $5.33 \pm 2.58$ & $0.44 \pm 0.34$ & $14.44 \pm 4.54$ \\
\hline Fight $(\mathrm{n})^{*,+}$ & $5.89 \pm 1.91$ & $40.78 \pm 8.42$ & $4.22 \pm 1.88$ \\
\hline Fight $(\mathrm{s})^{*,+}$ & $5.89 \pm 1.91$ & $48.89 \pm 10.08$ & $4.22 \pm 1.88$ \\
\hline Aggressive grooming $(\mathrm{n})^{*,+}$ & $0.78 \pm 0.36$ & $8.11 \pm 2.13$ & $0.67 \pm 0.37$ \\
\hline Aggressive grooming $(\mathrm{s})^{*,+}$ & $1.00 \pm 0.55$ & $28.67 \pm 9.30$ & $1.78 \pm 0.98$ \\
\hline Biting $(n)^{*,+}$ & $0.44 \pm 0.24$ & $19.33 \pm 3.20$ & $1.00 \pm 0.65$ \\
\hline Biting $(\mathrm{s})^{*,+}$ & $0.44 \pm 0.24$ & $30.44 \pm 6.89$ & $1.00 \pm 0.65$ \\
\hline
\end{tabular}

Data are presented as mean \pm standard deviation; $n$, number of the behavior; s, duration measured in seconds; CON, non-lead exposed offspring; LEAD, lead exposed on gestation and lactation period offspring; LEAD-CLZ, lead exposed on gestation and lactation with clozapine treatment on adolescence period; *Between CON and LEAD p-value $<0.05$; ${ }^{+}$Between LEAD and LEAD-CLZ p-value $<0.05$. 
luminescence kit (ELPIS-Biotech Inc., Daejeon, Korea).

\section{Immunohistochemistry}

Rats were deeply anesthetized with ethyl ether and perfused with $4 \%$ paraformaldehyde ( $\mathrm{N}=5-6$ animals/each group). The fixed brains were removed, frozen, and cut into $30-\mu \mathrm{m}$ thick sections for anatomical imaging of gene expression (6.24 mm interaural, $-2.76 \mathrm{~mm}$ bregma, section 10 of the Rat Brain Library). The expression levels were averaged to CA3 area of hippocampus on the same side of the brain. Frozen sections from the hippocam- pus were blocked with normal horse serum, incubated with antiDpysl2 (1:1,000; Atlas Antibodies AB, Stockholm, Sweden), Nefm (1:100; Cell Signaling Technology), and NeuN (1:100; Millipore) followed by incubation with Cy3-conjugated anti-rabbit and mouse secondary antibodies (1:500 and 1:800 Jackson ImmunoResearch Laboratories, Inc., West Grove, PA, USA). Fluorescent images were captured using a confocal laser-scanning microscope (FV10-ASW; Olympus, Tokyo, Japan). Images were quantified with ImageJ software using a protocol described previously with slight modifications [31,32].

Table 2. Behavior of non-lead exposed group, lead exposed group and lead exposure with clozapine treatment for adolescence in an open field test

\begin{tabular}{|c|c|c|c|}
\hline Actions & $\mathrm{CON}$ & LEAD & LEAD-CLZ \\
\hline Central entered ${ }^{*,+}$ & $18.29 \pm 3.81$ & $7.71 \pm 3.04$ & $17.86 \pm 3.39$ \\
\hline Line cross $^{*,+}$ & $7.57 \pm 3.46$ & $2.00 \pm 1.41$ & $8.71 \pm 3.09$ \\
\hline $\operatorname{Run}(\mathrm{n})^{*}$ & $0.71 \pm 0.48$ & $0.14 \pm 0.37$ & $0.14 \pm 0.37$ \\
\hline $\operatorname{Run}(\mathrm{s})^{*}$ & $1.00 \pm 0.81$ & $0.14 \pm 0.37$ & $0.14 \pm 0.37$ \\
\hline $\operatorname{Rear}(\mathrm{n})^{+}$ & $83.71 \pm 13.69$ & $73.14 \pm 12.81$ & $86.86 \pm 9.58$ \\
\hline Rear (s) & $180.43 \pm 12.23$ & $174.29 \pm 18.00$ & $172.57 \pm 25.48$ \\
\hline Grooming (n) & $11.00 \pm 4.83$ & $12.71 \pm 5.44$ & $15.29 \pm 9.50$ \\
\hline Grooming (s) & $132.86 \pm 28.66$ & $92.14 \pm 24.48$ & $129.86 \pm 13.39$ \\
\hline Cage sniff $(n)^{*,+}$ & $127.43 \pm 18.92$ & $95.29 \pm 16.07$ & $129.86 \pm 28.57$ \\
\hline Cage sniff $(\mathrm{s})^{*}$ & $407.29 \pm 28.97$ & $298.86 \pm 16.07$ & $371.43 \pm 28.40$ \\
\hline Immobile (n) & $1.42 \pm 1.99$ & $4.29 \pm 3.77$ & $1.14 \pm 2.04$ \\
\hline Immobile $(\mathrm{s})^{*,+}$ & $3.71 \pm 5.06$ & $16.28 \pm 13.69$ & $2.57 \pm 4.72$ \\
\hline
\end{tabular}

Data are presented as mean \pm standard deviation; $n$, number of the behavior; s, duration measured in seconds; CON, non-lead exposed offspring; LEAD, lead exposed on gestation and lactation period offspring; LEAD-CLZ, lead exposed on gestation and lactation with clozapine treatment on adolescence period; *Between CON and LEAD p-value $<0.05 ;{ }^{+}$Between LEAD and LEAD-CLZ p-value $<0.05$.

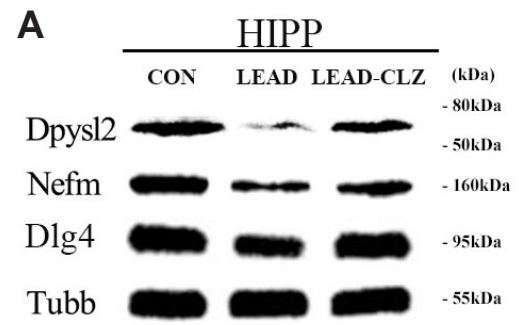

B

C

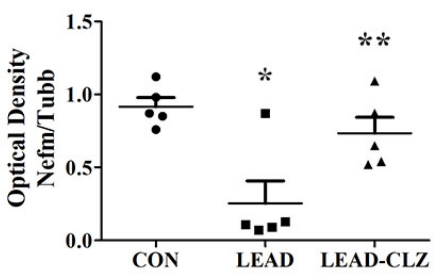

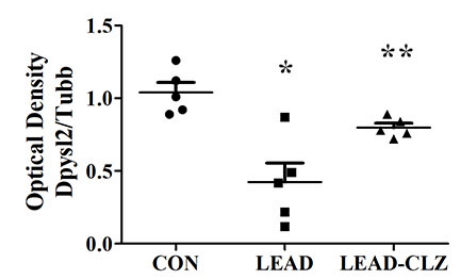

D

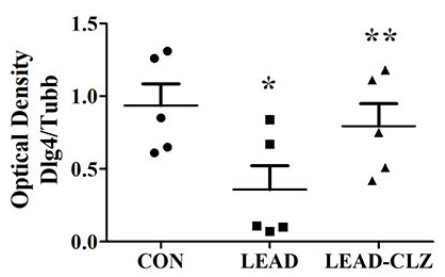

Fig. 2. Western blot analysis of Dpys/2, Nefm, and Dlg4 expression in brains of lead-exposed rats. (A) The expression of Dpysl2, Nefm, and Dlg4 was detected by western blot analysis. $\beta$-tubulin (Tubb) was used as an internal control. Lead-exposed rats demonstrated decreased hippocampal expression of Dpys|2, Nefm, and Dlg4 compared to control rats. Decreased expression of Dpysl2, Nefm and Dlg4 was restored ( $p<0.05$ ) by clozapine treatment. (B) The bar graph showing standard deviation (SD) ( $p<0.05$ compared to lead-exposed group). (C) The bar graph showing SD ( $<<$ 0.05 compared to lead-exposed group). (D) The bar graph showing SD ( $<0.05$ compared to lead-exposed group). HIPP, Hippocampus; LEAD, leadexposed offspring during prenatal and lactation periods; CON, non-lead exposed offspring; LEAD-CLZ, offspring exposed to lead and clozapine treatment. ${ }^{*} \mathrm{p}<0.05$ Between CON with LEAD; ** $\mathrm{p}<0.05$ Between LEAD with LEAD-CLZ. 


\section{Statistical analysis}

All data are expressed as mean \pm standard error of mean. They were compared using Student's t-test and/or ANOVA. If results of ANOVA indicated significant differences, a post-hoc analysis was performed with Tukey's honest significant difference (HSD) test. All statistical analyses were performed using IBM SPSS Statistics ver. 20 software (IBM Co., Armonk, NY, USA). Statistical significance was considered at $\mathrm{p}<0.05$.

\section{RESULTS}

\section{Prepulse inhibition}

Lead exposure during prenatal and lactation periods significantly affected sensorimotor gating. Results revealed significant differences in PPI levels among control, lead-exposed, and clozapine-treated groups. PPI levels at each stimulus level $(3,6$, and $12 \mathrm{~dB}$ above background) in the lead-exposed group were not significantly changed compared to those in the control group ( $p>$ 0.05 , Fig. $1 \mathrm{~A}$ ). However, the mean \% PPI at total prepulse stimulus level was significantly different between control and lead-exposed groups ( $p<0.05$, Fig. 1B). Clozapine treatment significantly increased the mean \% PPI level ( $p<0.05$, Fig. 1B).

\section{Social interaction test (SIT)}

All aggressive behavior scores during SIT were significantly increased in the offspring exposed to lead compared to those in the control group ( $p<0.05$, Table 1 ). Non-aggressive behavior scores of the lead-exposed offspring were decreased compared to those of the control group. Lead exposure also induced hyperactivity or excited states. The increased scores of aggressive behavior in the lead-exposed offspring were decreased to normal levels after clozapine treatment $(\mathrm{p}<0.05$, Table 1$)$, but not at each prepulse
A

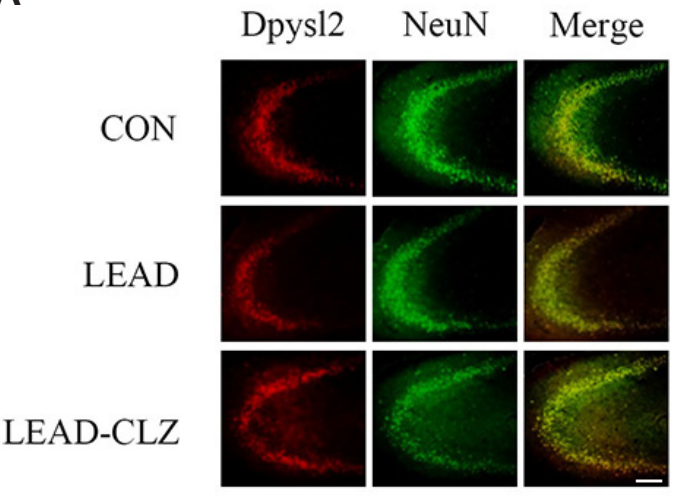

C

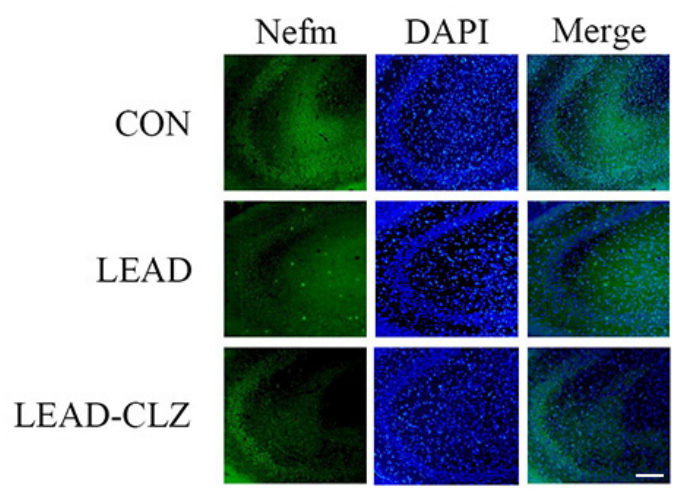

B

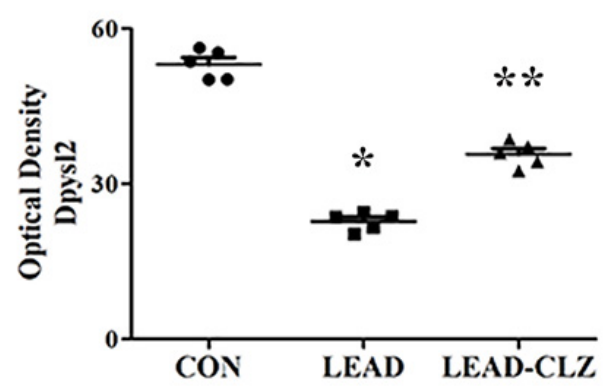

D

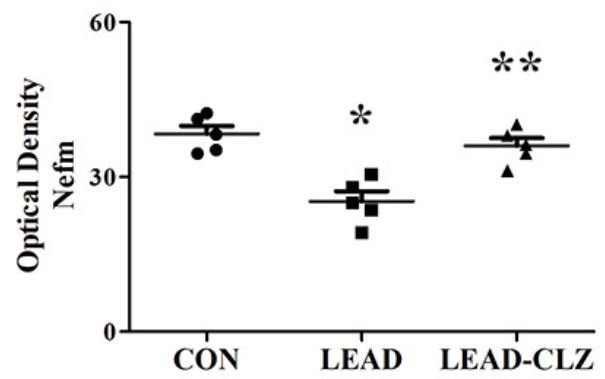

Fig. 3. Immunohistochemical analysis of Dpys/2 expression in the brains of lead-exposed rats. (A) Confocal microscopic image showing immunofluorescent staining of DpysI2 (anti-DpysI2, red, Cy3) with NeuN (anti-NeuN, green, FITC) in the hippocampus. Dpys/2 fluorescent staining intensity was decreased in these regions. Scale bar, $50 \mu \mathrm{m}$. (B) The bar graph showing standard deviation (SD) ( $p<0.05$ compared to lead-exposed group). (C) Confocal microscopic images showing immunofluorescent staining of Nefm (anti-Nefm, green, FITC) with DAPI (blue) in the hippocampus. The fluorescent staining intensity of Nefm was decreased in these regions. Scale bar, $50 \mu \mathrm{m}$. (D) The bar graph showing SD ( $p<0.05$ compared to the PNS group in the hippocampus). LEAD, lead-exposed offspring during prenatal and lactation periods; CON, non-lead-exposed offspring; LEAD-CLZ, offspring exposed to lead and treated with clozapine. ${ }^{*} p<0.05$ Between CON with LEAD; **p $<0.05$ Between LEAD with LEAD-CLZ. 
stimulus level ( $\mathrm{p}>0.05$, Fig. 1B).

\section{Open-field test}

The lead-exposed group significantly changed the number of central entries, line crossings, number and duration of rear, duration of cage sniff, and the degree of immobility compared to the control group. These scores were restored to near-normal levels after clozapine treatment $(\mathrm{p}<0.05$; Table 2$)$. However, the frequency of immobility behaviors was increased in the leadexposed group, but reduced to control levels after clozapine treatment $(\mathrm{p}<0.05$, Table 2$)$.

\section{Western blot and immunohistochemistry}

To investigate lead-induced changes in the expression of neurodevelopmental proteins such as Dpysl2, Dlg4, and Nefm, western blotting (Fig. 2) and immunohistochemical analyses (Fig. 3) of hippocampus areas from control, lead-exposed, and clozapinetreated offspring were performed. Western blot revealed that the expression of these proteins in the hippocampus of lead-exposed group was significantly decreased compared to those in the control ( $\mathrm{p}<0.05$, Fig. $2 \mathrm{~B}-\mathrm{D})$. These changes were restored by clozapine treatment ( $p<0.05$, Fig. $2 B-D)$. Dpysl2 and Nefm were differentially expressed in immunofluorescence-stained brain images among control, lead-exposed, and clozapine-treated groups (Fig. $3 \mathrm{~A}, \mathrm{C})$. The expression of Dpysl2 and Nefm proteins in the hippocampus of lead-exposed group was significantly decreased compared to that of the control (Fig. 3A, C). These changes were restored by clozapine treatment (Fig. 3A, C). The immunohistochemical staining intensity also varied significantly among the three groups ( $\mathrm{p}<0.05$, Fig. 3B, D).

\section{DISCUSSION}

In this study, impaired social interaction was observed in rat offspring exposed to lead. In social interaction test, the diminution of social interaction behavior reflected increased anxiety, hyperactivity, and aggressive behaviors in lead-exposed animal models. Lead-induced increases in aggressive behavior and hyperactivity were attenuated by clozapine treatment in the present study (Table 1). Additionally, a few behavioral patterns in open field test were reversed by clozapine treatment (Table 2). These results are consistent with previous studies showing that lead exposure may cause hyperactivity and deficits in learning and memory both in experimental animals and humans [31,33-35]. In PPI test, lead exposure failed to significantly affect the startle amplitude in a no-PPI trial in a previous study, but not startle amplitude at $120 \mathrm{~dB}$ [36]. Reduction in PPI of acoustic startle associated with chronic exposure to low-level oral lead might be attributed to lead-induced alteration in glutamate neurotransmission [37].
Clozapine appears to increase low PPI in normal subjects, consistent with a few studies investigating sustained treatment with atypical antipsychotics and schizophrenia [38]. Prenatal viral mimic-induced PPI disruption was normalized by pre-adolescent clozapine treatment. However, clozapine failed to significantly reduce \% PPI in adulthood, although it significantly reduced the mean \% PPI [38]. Our results also demonstrated that clozapine treatment significantly reduced the mean \% PPI level ( $\mathrm{p}<0.05$, Fig. 1B) without significantly affecting acute startle response ( $\mathrm{p}$ $>0.05$, Fig. 1A). These results suggested that our animal model was affected by low-level lead exposure probably due to inhibition of sensorimotor gating function caused by altered glutamate neurotransmission induced by NMDA antagonist. Several studies suggested that lead-induced neurotoxicity decreased the expression of NMDAR subtypes, and reduced the calcium-dependent synaptic transmission $[39,40]$.

We evaluated whether maternal lead exposure affected the expression of PSD95, Dpysl2, Lasp1, and Nefm. PSD95 is essential for post-synaptic connectivity, downstream signaling cascade, neuronal maturation, and plasticity $[41,42]$. The reduction in these proteins was expected to affect PSD95-NMDA receptor complex formation in postsynaptic membrane sites and related downstream signals, and alter brain development and cognition in lead-exposed dams. This speculation was consistent, at least partially, with previous reports showing that lead exposure during synaptogenesis led to NMDA receptor inhibition $[41,42]$. Furthermore, lead impaired slow axonal transport in rats with hyperphosphorylated neurofilaments, reducing the mobility of neurofilaments that are not extensively phosphorylated [43]. Our previous study has shown that NMDA antagonism may downregulate the expression of proteins such as Laspl in a schizophrenia-like animal model [44]. Several studies have reported that a schizophrenia-like model induced by prenatal stress showed altered expression of NMDA receptor as one of the glutamate receptors involved in neurodegeneration, learning, and memory $[45,46]$. Our recent study using another schizophrenia-like animal model showed that the expression of Dpysl2 was changed in prefrontal cortex and hippocampus area [23]. Therefore, proteins associated with brain development and cognition might be downregulated due to NMDA receptor inhibition caused by lead exposure. In the present study, our results indicated that decreased levels of brain development and cognition-related proteins can be restored by clozapine treatment. Clozapine is an effective partial agonist of muscarinic $\mathrm{M} 1$ receptors and an agonist of dopamine D1 receptors resulting in enhanced NMDA-R function [47]. Hence, clozapine may increase the NMDA-R transmission, explaining the unique efficacy of this agent. A recent study reported that the expression of SNPA-25, a synaptic gene, was influenced by clozapine treatment in male rats [47]. Although our data provided direct evidence suggesting that lead exposure decreased the levels of brain development and cognition-related proteins, the mechanism of decrease and recovery by clozapine treatment 
remains unclear. Therefore, further studies are needed to evaluate the role of clozapine for the treatment of lead exposure-induced neurodevelopmental psychiatric disorders such as autism and schizophrenia. In addition, cellular and animal models exposed to various levels of lead exposure are needed to determine the mechanism underlying the effect of clozapine.

\section{ACKNOWLEDGEMENTS}

The present study was performed with the support of Basic Science Research Program through the National Research Foundation of Korea (NRF) funded by the Ministry of Education (2016R1D1A1B03931619).

\section{CONFLICTS OF INTEREST}

The authors declare no conflicts of interest.

\section{REFERENCES}

1. Liu JT, Chen BY, Zhang JQ, Kuang F, Chen LW. Lead exposure induced microgliosis and astrogliosis in hippocampus of young mice potentially by triggering TLR4-MyD88-NFkB signaling cascades. Toxicol Lett. 2015;239:97-107.

2. Guilarte TR, Opler M, Pletnikov M. Is lead exposure in early life an environmental risk factor for Schizophrenia? neurobiological connections and testable hypotheses. Neurotoxicology. 2012;33:560-574.

3. Moreira EG, Vassilieff I, Vassilieff VS. Developmental lead exposure: behavioral alterations in the short and long term. Neurotoxicol Teratol. 2001;23:489-495.

4. Needleman HL, Gunnoe C, Leviton A, Reed R, Peresie H, Maher C, Barrett P. Deficits in psychologic and classroom performance of children with elevated dentine lead levels. $N$ Engl J Med. 1979;300:689-695.

5. Dietrich KN, Berger OG, Bhattacharya A. Symptomatic lead poisoning in infancy: a prospective case analysis. J Pediatr. 2000;137:568571.

6. Kaneshiro Olympio KP. Gonçalves C, Risso Günther WM, Henriques Bechara EJ. Neurotoxicity and aggressiveness triggered by low-level lead in children: a review. Rev Panam Salud Publica/Pan Am J Public Health. 2009;26:266-275.

7. Hu H, Téllez-Rojo MM, Bellinger D, Smith D, Ettinger AS, Lamadrid-Figueroa H, Schwartz J, Schnaas L, Mercado-García A, Hernández-Avila M. Fetal lead exposure at each stage of pregnancy as a predictor of infant mental development. Environ Health Perspect. 2006;114:1730-1735.

8. Budtz-Jørgensen E, Bellinger D, Lanphear B, Grandjean P; International Pooled Lead Study Investigators. An international pooled analysis for obtaining a benchmark dose for environmental lead exposure in children. Risk Anal. 2013;33:450-461.

9. Boksa P. Animal models of obstetric complications in relation to schizophrenia. Brain Res Brain Res Rev. 2004;45:1-17.
10. Kvajo M, McKellar H, Gogos JA. Avoiding mouse traps in schizophrenia genetics: lessons and promises from current and emerging mouse models. Neuroscience. 2012;211:136-164.

11. White LD, Cory-Slechta DA, Gilbert ME, Tiffany-Castiglioni E, Zawia NH, Virgolini M, Rossi-George A, Lasley SM, Qian YC, Basha MR. New and evolving concepts in the neurotoxicology of lead. Toxicol Appl Pharmacol. 2007;225:1-27.

12. Lasley SM, Gilbert ME. Glutamatergic components underlying leadinduced impairments in hippocampal synaptic plasticity. Neurotoxicology. 2000;21:1057-1068.

13. Brini M, Calì T, Ottolini D, Carafoli E. Neuronal calcium signaling: function and dysfunction. Cell Mol Life Sci. 2014;71:2787-2814.

14. Rao Barkur R, Bairy LK. Evaluation of passive avoidance learning and spatial memory in rats exposed to low levels of lead during specific periods of early brain development. Int J Occup Med Environ Health. 2015;28:533-544.

15. Lanphear BP, Dietrich K, Auinger P, Cox C. Cognitive deficits associated with blood lead concentrations $<10$ microg/dL in US children and adolescents. Public Health Rep. 2000;115:521-529.

16. Braff D, Stone C, Callaway E, Geyer M, Glick I, Bali L. Prestimulus effects on human startle reflex in normals and schizophrenics. Psychophysiology. 1978;15:339-343.

17. Braff DL, Grillon C, Geyer MA. Gating and habituation of the startle reflex in schizophrenic patients. Arch Gen Psychiatry. 1992; 49:206-215.

18. Geyer MA. The family of sensorimotor gating disorders: comorbidities or diagnostic overlaps? Neurotox Res. 2006;10:211-220.

19. Pouzet B, Andersen MP, Hogg S. Effects of acute treatment with antidepressant drugs on sensorimotor gating deficits in rats. Psychopharmacology (Berl). 2005;178:9-16.

20. Le Pen G, Moreau JL. Disruption of prepulse inhibition of startle reflex in a neurodevelopmental model of schizophrenia: reversal by clozapine, olanzapine and risperidone but not by haloperidol. Neuropsychopharmacology. 2002;27:1-11.

21. Khan AH, Zaidi S. Clozapine: improvement of negative symptoms of schizophrenia. Cureus. 2017;9:e1973.

22. Won HS, Kim YO, Lee HY, Im JY, Lee SH, Cho IH, Lee SW, Park CG, Kim HK, Kwon JT, Kim HJ. Effect of valeriana fauriei extract on the neurodevelopmental proteins expression and behavioral patterns in maternal immune activation animal model. Korean J Med Crop Sci. 2016;24:341-350.

23. O'Shea PJ, Harvey CB, Suzuki H, Kaneshige M, Kaneshige K, Cheng SY, Williams GR. A thyrotoxic skeletal phenotype of advanced bone formation in mice with resistance to thyroid hormone. Mol Endocrinol. 2003;17:1410-1424.

24. Bradshaw NJ, Porteous DJ. DISC1-binding proteins in neural development, signalling and schizophrenia. Neuropharmacology. 2012;62:1230-1241.

25. Taya S, Shinoda T, Tsuboi D, Asaki J, Nagai K, Hikita T, Kuroda S, Kuroda K, Shimizu M, Hirotsune S, Iwamatsu A, Kaibuchi K. DISC1 Regulates the Transport of the NUDEL/LIS1/14-3-3ع Complex through Kinesin-1. J Neurosci. 2000;27:15-26.

26. Soares DC, Carlyle BC, Bradshaw NJ, Porteous DJ. DISC1: Structure, Function, and Therapeutic Potential for Major Mental Illness. ACS Chem Neurosci. 2011;2:609-632.

27. Shinoda T, Taya S, Tsuboi D, Hikita T, Matsuzawa R, Kuroda S, Iwamatsu A, bKaibuchi K. DISC1 regulates neurotrophin-induced axon 
elongation via interaction with Grb2. J Neurosci. 2007;27:4-14.

28. Institute of Laboratory Animal Resources (Estats Units d'Amèrica). Guide for the care and use of laboratory animals. Washington: National Academy Press; 1996. p. xii-125.

29. Schroeder M, Sultany T, Weller A. Prenatal stress effects on emotion regulation differ by genotype and sex in prepubertal rats. Dev Psychobiol. 2013;55:176-192.

30. Lee H, Won H, Im J, Kim YO, Lee S, Cho IH, Kim HK, Kwon JT, Kim HJ. Effect of Valeriana fauriei extract on the offspring of adult rats exposed to prenatal stress. Int J Mol Med. 2016;38:251-258.

31. Jung HY, Chung TH, Hwang IK. Dendropanax morbifera Léveille extract ameliorates memory impairments and inflammatory responses in the hippocampus of streptozotocin-induced type 1 diabetic rats. Mol Cell Toxicol. 2016;12:429-436.

32. Altmann L, Weinsberg F, Sveinsson K, Lilienthal H, Wiegand $H$, Winneke G. Impairment of long-term potentiation and learning following chronic lead exposure. Toxicol Lett. 1993;66:105-112.

33. Chen HH, Ma T, Paul IA, Spencer JL, Ho IK. Developmental lead exposure and two-way active avoidance training alter the distribution of protein kinase $\mathrm{C}$ activity in the rat hippocampus. Neurochem Res. 1997;22:1119-1125.

34. Ma T, Chen HH, Ho IK. Effects of chronic lead $(\mathrm{Pb})$ exposure on neurobehavioral function and dopaminergic neurotransmitter receptors in rats. Toxicol Lett. 1999;105:111-121.

35. Commissaris RL, Tavakoli-Nezhad M, Barron AJ, Pitts DK. Effects of chronic low-level oral lead exposure on prepulse inhibition of acoustic startle in the rat. Neurotoxicol Teratol. 2000;22:55-60.

36. Vollenweider FX, Barro M, Csomor PA, Feldon J. Clozapine enhances prepulse inhibition in healthy humans with low but not with high prepulse inhibition levels. Biol Psychiatry. 2006;60:597-603.

37. Kumari V, Soni W, Sharma T. Normalization of information processing deficits in schizophrenia with clozapine. Am J Psychiatry. 1999;156:1046-1051.

38. Xu J, Yan HC, Yang B, Tong LS, Zou YX, Tian Y. Effects of lead exposure on hippocampal metabotropic glutamate receptor subtype 3 and 7 in developmental rats. J Negat Results Biomed. 2009;8:5.
39. Neal AP, Worley PF, Guilarte TR. Lead exposure during synaptogenesis alters NMDA receptor targeting via NMDA receptor inhibition. Neurotoxicology. 2011;32:281-289.

40. Hossain S, Bhowmick S, Jahan S, Rozario L, Sarkar M, Islam S, Basunia MA, Rahman A, Choudhury BK, Shahjalal H. Maternal lead exposure decreases the levels of brain development and cognitionrelated proteins with concomitant upsurges of oxidative stress, inflammatory response and apoptosis in the offspring rats. Neurotoxicology. 2016;56:150-158.

41. Yokoyama K, Araki S. Assessment of slow axonal transport in leadexposed rats. Environ Res. 1992;59:440-446.

42. Joo J, Lee S, Nah SS, Kim YO, Kim DS, Shim SH, Hwangbo Y, Kim HK, Kwon JT, Kim JW, Song HY, Kim HJ. Laspl is down-regulated in NMDA receptor antagonist-treated mice and implicated in human schizophrenia susceptibility. J Psychiatr Res. 2013;47:105-112.

43. Calabrese F, Guidotti G, Molteni R, Racagni G, Mancini M, Riva MA. Stress-induced changes of hippocampal NMDA receptors: modulation by duloxetine treatment. PLoS One. 2012;7:e37916.

44. Rubio MD, Drummond JB, Meador-Woodruff JH. Glutamate receptor abnormalities in schizophrenia: implications for innovative treatments. Biomol Ther (Seoul). 2012;20:1-18.

45. Lee H, Joo J, Nah SS, Kim JW, Kim HK, Kwon JT, Lee HY, Kim YO, Kim HJ. Changes in Dpysl2 expression are associated with prenatally stressed rat offspring and susceptibility to schizophrenia in humans. Int J Mol Med. 2015;35:1574-1586.

46. Sur C, Mallorga PJ, Wittmann M, Jacobson MA, Pascarella D, Williams JB, Brandish PE, Pettibone DJ, Scolnick EM, Conn PJ. Ndesmethylclozapine, an allosteric agonist at muscarinic 1 receptor, potentiates N-methyl-D-aspartate receptor activity. Proc Natl Acad Sci U S A. 2003;100:13674-13679.

47. von Wilmsdorff M, Manthey F, Bouvier ML, Staehlin O, Falkai P, Meisenzahl-Lechner E, Schmitt A, Gebicke-Haerter PJ. Effects of haloperidol and clozapine on synapse-related gene expression in specific brain regions of male rats. Eur Arch Psychiatry Clin Neurosci. 2018;268:555-563. 